\title{
Pullback Random Attractors for Non-Autonomous Stochastic Fractional FitzHugh-Nagumo System
}

\author{
Chunxiao Guo ${ }^{1}$, Yiju Chen ${ }^{*}$, Yanfeng Guo ${ }^{2,3}$ \\ ${ }^{1}$ Department of Mathematics, China University of Mining and Technology Beijing, Beijing, China \\ ${ }^{2}$ School of Mathematics and Physics, China University of Geosciences, Wuhan, China \\ ${ }^{3}$ School of Science, Guangxi University of Science and Technology, Liuzhou, China \\ Email: *yijuchen6@163.com
}

How to cite this paper: Guo, C.X., Chen, Y.J. and Guo, Y.F. (2020) Pullback Random Attractors for Non-Autonomous Stochastic Fractional FitzHugh-Nagumo System. Journal of Applied Mathematics and Physics, 8, 115-131.

https://doi.org/10.4236/jamp.2020.81010

Received: December 6, 2019

Accepted: January 5, 2020

Published: January 8, 2020

Copyright $\odot 2020$ by author(s) and Scientific Research Publishing Inc. This work is licensed under the Creative Commons Attribution International License (CC BY 4.0).

http://creativecommons.org/licenses/by/4.0/

\begin{abstract}
This paper is concerned with the asymptotic behavior of solutions for a class of non-autonomous fractional FitzHugh-Nagumo equations deriven by additive white noise. We first provide some sufficient conditions for the existence and uniqueness of solutions, and then prove the existence and uniqueness of tempered pullback random attractors for the random dynamical system generated by the solutions of considered equations in an appropriate Hilbert space. The proof is based on the uniform estimates and the decomposition of dynamical system.
\end{abstract}

\section{Keywords}

Non-Autonomous Stochastic Fractional FitzHugh-Nagumo System, Random Attractor, Additive White Noise

\section{Introduction}

In this paper, we investigate the random attractor of the non-autonomous stochastic fractional FitzHugh-Nagumo equations with additive white noise in bounded domains. Let $s \in(0,1), \tau \in \mathbf{R}$ and $\mathbf{U}$ be a smooth bounded domain of $\mathbf{R}^{n}$. We consider the following stochastic system in $U$ :

$$
\begin{gathered}
\mathrm{d} \tilde{u}+\left((-\Delta)^{s} \tilde{u}+\lambda_{1} \tilde{u}+\alpha_{1} \tilde{v}\right) \mathrm{d} t=f(x, \tilde{u}) \mathrm{d} t+g(t, x) \mathrm{d} t+\phi_{1}(x) \mathrm{d} \omega_{1}, x \in \mathbf{U}, t>\tau, \\
\mathrm{d} \tilde{v}+\left(\lambda_{2} \tilde{v}-\alpha_{2} \tilde{u}\right) \mathrm{d} t=h(t, x) \mathrm{d} t+\phi_{2}(x) \mathrm{d} \omega_{2}, x \in \mathbf{U}, t>\tau,
\end{gathered}
$$

with initial conditions 


$$
\tilde{u}(\tau, x)=\tilde{u}_{\tau}(x), \tilde{v}(\tau, x)=\tilde{v}_{\tau}(x), x \in \mathbf{U},
$$

and boundary conditions

$$
\tilde{u}(t, x)=\tilde{v}(t, x)=0, x \in \partial \mathbf{U}, t>\tau,
$$

where $\tilde{u}=\tilde{u}(t, x), \tilde{v}=\tilde{v}(t, x)$ are real-valued functions on $[\tau,+\infty) \times \mathbf{U}$, $\lambda_{1}, \lambda_{2}, \alpha_{1}$ and $\alpha_{2}$ are positive constants, $g(t, x) \in L_{l o c}^{2}\left(\mathbf{R}, L^{2}(\mathbf{U})\right)$, $h(t, x) \in L_{l o c}^{2}\left(\mathbf{R}, H^{s}(\mathbf{U})\right), \quad \phi_{1} \in D\left((-\Delta)^{s}\right) \cap W^{2 s, p}(\mathbf{U}) \quad(p \geq 1$ and the details of these spaces will be given later $), \phi_{2} \in L^{2}(\mathbf{U}), W_{1}\left(\cdot, \omega_{1}\right)$ and $W_{2}\left(\cdot, \omega_{2}\right)$ are independent two-sided real-valued Wiener processes with the standard process $\omega(\cdot):=\left(\omega_{1}(\cdot), \omega_{2}(\cdot)\right)$ on a probability space which will be specified below. The nonlinear function $f(x, \tilde{u})$ is a differential function about two variables satisfying: for all $(x, s) \in \mathbf{U} \times \mathbf{R}$ and $\beta_{i}>0, \quad(i=1,2)$

$$
\begin{gathered}
f(x, s) s \leq-\beta_{1}|s|^{p}+\psi_{1}(x), \\
|f(x, s)| \leq \beta_{2}|s|^{p-1}+\psi_{2}(x), \\
\frac{\partial f}{\partial s}(x, s) \leq \psi_{3}(x), \\
|f(x, s)-f(y, s)| \leq\left|\psi_{4}(x)-\psi_{4}(y)\right|,
\end{gathered}
$$

where $p \geq 2$ are constant, $\psi_{1} \in L^{1}(\mathbf{U}), \psi_{2} \in L^{q}(\mathbf{U})$ with $\frac{1}{p}+\frac{1}{q}=1$, and $\psi_{3} \in L^{\infty}(\mathbf{U}), \psi_{4} \in H^{s}(\mathbf{U})$.

The FitzHugh-Nagumo system is a model for describing the signal transmission across axons in neurobiology, see [1] [2] [3]. The long term dynamics and inertial manifolds for the deterministic FitzHugh-Nagumo system have been extensively studied by many authors, see [4] [5] [6]. The existence of random attractor for the stochastic or lattice FitzHugh-Nagumo system has been investigated in [7] [8] [9] [10]. Recently, the fractional FitzHugh-Nagumo monodomain model is presented, the model consists of a coupled fractional nonlinear reaction-diffusion model and a system of ordinary differential equations. The stability and convergence are discussed using numerical method in [11]. To the best of our knowledge, there are some results on numerical calculation of deterministic fractional FitzHugh-Nagumo equation, but few results for theory study of stochastic fractional FitzHugh-Nagumo equation, especially for $s \in\left(0, \frac{1}{2}\right]$. As far as the author is aware, the attractors of the fractional stochastic equations are not well studied, it seems that the only publications [12] [13] in this respect, where the authors researched the existence of random attractors for the fractional stochastic equation with $s \in\left(\frac{1}{2}, 1\right)$. In [14] [15], the authors discussed the asymptotic behavior of fractional reaction-diffusion equation with $s \in(0,1)$.

In this paper, we explore the long time behavior of the solutions when the Equations (1)-(4) are perturbed by an additive white noise. There are several dif- 
ficulties in this paper. Firstly, since the FitzHugh-Nagumo equation is a coupling equations, thus the uniform estimates of solutions are slightly different from the reaction-diffusion equation [15]. On the other hand, comparing with [15], we are concerned with the existence of random attractors of the fractional FitzHugh-Nagumo equation on $U$ driven by additive noise rather than multiplicative noise, so new difficulties arise from the estimates for some terms, especially the nonlinearity $f$. Finally, the lack of higher regularity of the solution $\tilde{v}$ for the problem (2) which makes the difficulty to construct a compact attracting set of the random dynamical system. To achieve our goals, we must overcome these difficulties and establish the pullback asymptotic compactness of solutions in $L^{2}(\mathbf{U}) \times L^{2}(\mathbf{U})$. We decompose the second component $\tilde{v}$ into a sum of two parts to overcome the lack of higher regularity like dealing with the wave equation in [16] [17].

This paper is organized as follows. In Section 2, we recall some basic concepts and define a continuous random dynamical system based on the solutions of the stochastic fractional FitzHugh-Nagumo Equations (1)-(4) in $L^{2}(\mathbf{U}) \times L^{2}(\mathbf{U})$. We derive some uniform estimates for solutions and prove the existence of a pullback random attractor by pullback asymptotic compactness of solutions in Section 3 .

\section{Cocycles of the Stochastic Fractional FitzHugh-Nagumo System}

In this section, we first collect some well-known results from the theory of random attractors and non-autonomous random dynamical systems. For further details, readers are also referred to [18] [19] [20] [21].

Let $\left(\Omega, \mathcal{F}, P,\left(\theta_{t}\right)_{t \in \mathbf{R}}\right)$ be a parametric dynamical system on the probability space $(\Omega, \mathcal{F}, P)$, in which $\Omega=\left\{\omega=\left(\omega_{1}, \omega_{2}\right) \in C\left(\mathbf{R}, \mathbf{R}^{2}\right): \omega(0)=0\right\}, \mathcal{F} \quad$ is the Borel $\sigma$-algebra induced by the compact-open topology of $\Omega$ and $P$ is the Wiener measure on $(\Omega, \mathcal{F})$, the group $\theta_{t}: \Omega \rightarrow \Omega$ defined by $\theta_{t} \omega(\cdot)=\omega(\cdot+t)-\omega(t)$ for $t \in \mathbf{R}, \omega \in \Omega$. We usually write the norm of $L^{2}\left(\mathbf{R}^{n}\right)$ as $\|\cdot\|$ and the scalar product of $L^{2}\left(\mathbf{R}^{n}\right)$ as $(\cdot, \cdot)$. We also use $\|\cdot\|_{X}$ to denote the norm of a Banach space $X$.

In the following, "property holds for a.e. $\omega \in \Omega$ with respect to $\left(\theta_{t}\right)_{t \in \mathbf{R}}$ " means that there is $\tilde{\Omega} \subset \Omega$ with $P(\tilde{\Omega})=1$ and $\theta_{t} \tilde{\Omega}=\tilde{\Omega}$ for all $t \in \mathbf{R}$ such that property holds for all $\omega \in \tilde{\Omega}$.

Definition 2.1. Let $\mathcal{D}$ be a collection of some families of nonempty subset of $X$ and $\Phi$ a continuous cocycle on $X,\{\mathcal{A}(\tau, \omega)\}_{\omega \in \Omega} \in \mathcal{D}$. Then $\{\mathcal{A}(\tau, \omega)\}_{\omega \in \Omega}$ is called a $\mathcal{D}$-pullback random attractor for $\Phi$ if the following conditions are satisfied, for every $\tau \in \mathbf{R}$ and a.e. $\omega \in \Omega$,

1) $\{\mathcal{A}(\tau, \omega)\}$ is compact in $X$, and $\omega \rightarrow d_{X}(x, \mathcal{A}(\tau, \omega))$ is measurable;

2) $\{\mathcal{A}(\tau, \omega)\}$ is strictly invariant, i.e., $\Phi(t, \tau, \omega, \mathcal{A}(\tau, \omega))=\mathcal{A}\left(t+\tau, \theta_{t} \omega\right)$, for all $t \geq 0$;

3) $\{\mathcal{A}(\tau, \omega)\}$ attracts every member of $\mathcal{D}$ in $X$, i.e., for all $B \in \mathcal{D}$, we have 


$$
\lim _{t \rightarrow+\infty} d_{X}\left(\Phi\left(t, \tau-t, \theta_{-t} \omega, B\left(\tau-t, \theta_{-t} \omega\right)\right), \mathcal{A}(\tau, \omega)\right)=0,
$$

where $d_{X}$ is the Hausdorff semi-distance in $X$.

Proposition 2.1. Suppose $X$ is a separable Banach spaces. Let $\mathcal{D}$ be an inclusion-closed collection of some families of nonempty subsets of $X$ and $\Phi$ be a continuous cocycle on $X$ over $\left(\Omega, \mathcal{F}, P,\left(\theta_{t}\right)_{t \in \mathbf{R}}\right)$. For all $t \in \mathbf{R}^{+}, \tau \in \mathbf{R}$, and $\omega \in \Omega, \Phi$ has a unique $\mathcal{D}$-pullback random attractor $\mathcal{A}(\tau, \omega)$ in $\mathcal{D}$ given by,

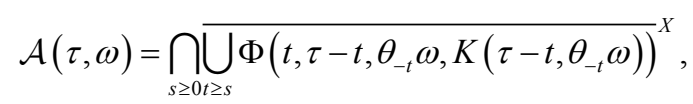

if 1) $\Phi$ has a compact measurable $\mathcal{D}$-pullback absorbing set $K$ in $\mathcal{D}$.

2) $\Phi$ is $\mathcal{D}$-pullback asymptotically compact in $X$.

To describe the main results of this raper, we review some concepts of the fractional Laplace operator on the bounded domain $\mathbf{U}$ (see [22] for details). Let $\mathcal{S}$ be the Schwartz space of rapidly decaying $C^{\infty}$ functions on $\mathbf{R}^{n}$, then for $0<s<1$, the fractional Laplace operator $(-\Delta)^{s}$ is given by, for $u \in \mathcal{S}$, $x \in \mathbf{R}^{n}$,

$$
(-\Delta)^{s} u(x)=-\frac{1}{2} C(n, s) P . V \cdot \int_{\mathbf{R}^{n}} \frac{u(x+y)+u(x-y)-2 u(x)}{|y|^{n+2 s}} \mathrm{~d} y,
$$

where $C(n, s)$ is a positive constant.

Let $H^{s}\left(\mathbf{R}^{n}\right)$ be the fractional Sobolev space defined by

$$
H^{s}\left(\mathbf{R}^{n}\right)=\left\{u \in L^{2}\left(\mathbf{R}^{n}\right): \int_{\mathbf{R}^{n}} \int_{\mathbf{R}^{n}} \frac{|u(x)-u(y)|^{2}}{|x-y|^{n+2 s}} \mathrm{~d} x \mathrm{~d} y<\infty\right\},
$$

which is equipped with the norm

$$
\|u\|_{H^{s}\left(\mathbf{R}^{n}\right)}=\left(\int_{\mathbf{R}^{n}}|u(x)|^{2} \mathrm{~d} x+\int_{\mathbf{R}^{n}} \int_{\mathbf{R}^{n}} \frac{|u(x)-u(y)|^{2}}{|x-y|^{n+2 s}} \mathrm{~d} x \mathrm{~d} y\right)^{\frac{1}{2}} .
$$

Note that $H^{s}\left(\mathbf{R}^{n}\right)$ is a Hilbert space with inner product given by

$$
\begin{aligned}
& (u, v)_{H^{s}\left(\mathbf{R}^{n}\right)}=\int_{\mathbf{R}^{n}} u(x) v(x) \mathrm{d} x \mathrm{~d} y \\
& +\int_{\mathbf{R}^{n}} \int_{\mathbf{R}^{n}} \frac{(u(x)-u(y))(v(x)-v(y))}{|x-y|^{n+2 s}} \mathrm{~d} x \mathrm{~d} y, u, v \in H^{s}\left(\mathbf{R}^{n}\right) .
\end{aligned}
$$

For convenience, we will also use the notation:

$$
\|u\|_{\dot{H}^{s}\left(\mathbf{R}^{n}\right)}^{2}=\int_{\mathbf{R}^{n}} \int_{\mathbf{R}^{n}} \frac{|u(x)-u(y)|^{2}}{|x-y|^{n+2 s}} \mathrm{~d} x \mathrm{~d} y, u \in H^{s}\left(\mathbf{R}^{n}\right) .
$$

By proposition 3.6 in [22], we find that:

$$
\|u\|_{H^{s}\left(\mathbf{R}^{n}\right)}^{2}=\|u\|_{L^{2}\left(\mathbf{R}^{n}\right)}^{2}+\frac{2}{C(n, s)}\left\|(-\Delta)^{\frac{s}{2}} u\right\|_{L^{2}\left(\mathbf{R}^{n}\right)}^{2} \text {, for all } u \in H^{s}\left(\mathbf{R}^{n}\right),
$$


and hence $\left(\|u\|_{L^{2}\left(\mathbf{R}^{n}\right)}^{2}+\left\|(-\Delta)^{\frac{s}{2}} u\right\|_{L^{2}\left(\mathbf{R}^{n}\right)}^{2}\right)^{\frac{1}{2}}$ is an equivalent norm of $H^{s}\left(\mathbf{R}^{n}\right)$. Similar to $H^{s}\left(\mathbf{R}^{n}\right)$, we can define $H^{2 s}\left(\mathbf{R}^{n}\right)$ and $W^{2 s, p}\left(\mathbf{R}^{n}\right)$ for $s \in(0,1)$ (see [22]).

Since the fractional operator $(-\Delta)^{s}$ is non-local, we here interpret the boundary (4) as $\tilde{u}(t, x)=\tilde{v}(t, x)=0, x \in \mathbf{R}^{n} \backslash \mathbf{U}$ instead of $\tilde{u}(t, x)=\tilde{v}(t, x)=0, x \in \partial \mathbf{U}$. From [23] and the references therein, we know such a interpretation is consistent with the non-local nature of the fractional operator. Thus, let $H=\left\{(u, v) \in L^{2}\left(\mathbf{R}^{n}\right) \times L^{2}\left(\mathbf{R}^{n}\right): u=v=0\right.$ a.e. on $\left.\mathbf{R}^{n} \backslash \mathbf{U}\right\}$ and $V=\left\{(u, v) \in H^{s}\left(\mathbf{R}^{n}\right) \times H^{s}\left(\mathbf{R}^{n}\right): u=v=0\right.$ a.e. on $\left.\mathbf{R}^{n} \backslash \mathbf{U}\right\}$.

Then, we give the essential assumptions and define a continuous random dynamical system for the stochastic fractional FitzHugh-Nagumo equations in $H$. More precisely, we consider:

$$
\begin{gathered}
\mathrm{d} \tilde{u}+\left((-\Delta)^{s} \tilde{u}+\lambda_{1} \tilde{u}+\alpha_{1} \tilde{v}\right) \mathrm{d} t \\
=f(x, \tilde{u}) \mathrm{d} t+g(t, x) \mathrm{d} t+\phi_{1}(x) \mathrm{d} \omega_{1}, x \in \mathbf{U}, t>\tau, \\
\mathrm{d} \tilde{v}+\left(\lambda_{2} \tilde{v}-\alpha_{2} \tilde{u}\right) \mathrm{d} t=h(t, x) \mathrm{d} t+\phi_{2}(x) \mathrm{d} \omega_{2}, x \in \mathbf{U}, t>\tau,
\end{gathered}
$$

with initial conditions

$$
\tilde{u}(\tau, x)=\tilde{u}_{\tau}(x), \tilde{v}(\tau, x)=\tilde{v}_{\tau}(x), x \in \mathbf{U},
$$

and boundary conditions

$$
\tilde{u}(t, x)=\tilde{v}(t, x)=0, x \in \mathbf{R}^{n} \backslash \mathbf{U}, t>\tau .
$$

Define $z(\omega):=\left(z_{1}\left(\omega_{1}\right), z_{2}\left(\omega_{2}\right)\right)$ by

$$
\begin{aligned}
& z_{1}\left(\omega_{1}\right)=-\lambda_{1} \int_{-\infty}^{0} \mathrm{e}^{\lambda_{1} s} \omega_{1}(s) \mathrm{d} s, \\
& z_{2}\left(\omega_{2}\right)=-\lambda_{2} \int_{-\infty}^{0} \mathrm{e}^{\lambda_{2} s} \omega_{2}(s) \mathrm{d} s .
\end{aligned}
$$

It is well know that $y_{i}(t, \omega)=z_{i}\left(\theta_{t} \omega_{i}\right)(i=1,2)$ is the unique stationary solution of the following stochastic equations

$$
\begin{aligned}
& \mathrm{d} y_{1}+\lambda_{1} y_{1} \mathrm{~d} t=\mathrm{d} W_{1}(t), \\
& \mathrm{d} y_{2}+\lambda_{2} y_{2} \mathrm{~d} t=\mathrm{d} W_{2}(t) .
\end{aligned}
$$

In addition, assume that $\delta=\min \left\{\lambda_{1}, \lambda_{2}\right\}$, from [18], we have

$$
\int_{-\infty}^{0} \mathrm{e}^{\delta s}\left(\left|z_{1}\left(\theta_{s} \omega_{1}\right)\right|^{2}+\left|z_{1}\left(\theta_{s} \omega_{1}\right)\right|^{p}+\left|z_{1}\left(\theta_{s} \omega_{1}\right)\right|^{2 p-2}\right) \mathrm{d} s<\infty,
$$

and

$$
\int_{-\infty}^{0} \mathrm{e}^{\delta s}\left|z_{2}\left(\theta_{s} \omega_{2}\right)\right|^{2} \mathrm{~d} s<\infty
$$

It follows from [10] that there exists a $\theta_{t}$-invariant set of full measure (still denoted by $\Omega$ ) such that $z_{i}\left(\theta_{t} \omega_{i}\right)(i=1,2)$ are both continuous in $t$ for each $\omega \in \Omega$.

We now transform the stochastic Equations (11)-(14) into a pathwise determinis- 
tic one by using the random variable $z_{i}(i=1,2)$. Given $\tau \in \mathbf{R}, t \geq \tau, \omega \in \Omega$ and $\left(\tilde{u}_{\tau}, \tilde{v}_{\tau}\right) \in L^{2}(\mathbf{U}) \times L^{2}(\mathbf{U})$, let $(\tilde{u}, \tilde{v})=\left(\tilde{u}\left(t, \tau, \omega, \tilde{u}_{\tau}\right), \tilde{v}\left(t, \tau, \omega, \tilde{v}_{\tau}\right)\right)$ is a solution of (11)-(14), introduce variables transformation:

$$
\begin{aligned}
u\left(t, \tau, \omega, u_{\tau}\right) & :=\tilde{u}\left(t, \tau, \omega, \tilde{u}_{\tau}\right)-\phi_{1} z_{1}\left(\theta_{t} \omega_{1}\right) \text { with } u_{\tau}=\tilde{u}_{\tau}-\phi_{1} z_{1}\left(\theta_{\tau} \omega_{1}\right), \\
v\left(t, \tau, \omega, v_{\tau}\right) & :=\tilde{v}\left(t, \tau, \omega, \tilde{v}_{\tau}\right)-\phi_{2} z_{2}\left(\theta_{t} \omega_{2}\right) \text { with } v_{\tau}=\tilde{v}_{\tau}-\phi_{2} z_{2}\left(\theta_{\tau} \omega_{2}\right) .
\end{aligned}
$$

By (11)-(14) and (15)-(16) we get

$$
\begin{aligned}
& \frac{\partial u}{\partial t}+(-\Delta)^{s} u+\lambda_{1} u+\alpha_{1} v \\
& =f(x, \tilde{u})+g(t, x)-(-\Delta)^{s} \phi_{1} z_{1}\left(\theta_{t} \omega_{1}\right)-\alpha_{1} \phi_{2} z_{2}\left(\theta_{t} \omega_{2}\right), x \in \mathbf{U}, t>\tau, \\
& \quad \frac{\partial v}{\partial t}+\lambda_{2} v-\alpha_{2} u=h(t, x)+\alpha_{2} \phi_{1} z_{1}\left(\theta_{t} \omega_{1}\right), x \in \mathbf{U}, t>\tau,
\end{aligned}
$$

with initial conditions

$$
u(\tau, x)=u_{\tau}(x), v(\tau, x)=v_{\tau}(x), x \in \mathbf{U},
$$

and boundary conditions

$$
u(t, x)=v(t, x)=0, x \in \mathbf{R}^{n} \backslash \mathbf{U}, t>\tau .
$$

Recall that $H$ and $V$ are Hilbert space. Note that the definition of $H$ is consistent with conditions (23) and (24), since $(u, v) \in L^{2}(\mathbf{U}) \times L^{2}(\mathbf{U})$ can be considered as an element of $H$ by setting $u(x)=v(x)=0$ for $x \in \mathbf{R}^{n} \backslash \mathbf{U}$. It follows from the standard arguments as in [24] that under conditions (1)-(7), problem (21)-(24) is well-posed in $H$. The unique solution defines two cocycles $\Phi, \tilde{\Phi}: \mathbf{R}^{+} \times \mathbf{R} \times \Omega \times H \rightarrow H$ as follows: for $t \in \mathbf{R}^{+}, \tau \in \mathbf{R}, \omega \in \Omega$ and $\left(u_{\tau}, v_{\tau}\right) \in H$

$$
\begin{gathered}
\Phi\left(t, \tau, \omega,\left(u_{\tau}, v_{\tau}\right)\right)=\left(u\left(t+\tau, \tau, \theta_{-\tau} \omega, u_{\tau}\right), v\left(t+\tau, \tau, \theta_{-\tau} \omega, v_{\tau}\right)\right), \\
\tilde{\Phi}\left(t, \tau, \omega,\left(\tilde{u}_{\tau}, \tilde{v}_{\tau}\right)\right)=\left(\tilde{u}\left(t+\tau, \tau, \theta_{-\tau} \omega, \tilde{u}_{\tau}\right), \tilde{v}\left(t+\tau, \tau, \theta_{-\tau} \omega, \tilde{v}_{\tau}\right)\right) \\
=\Phi\left(t, \tau, \omega,\left(u_{\tau}, v_{\tau}\right)\right)+\left(\phi_{1} z_{1}\left(\theta_{t} \omega_{1}\right), \phi_{2} z_{2}\left(\theta_{t} \omega_{2}\right)\right),
\end{gathered}
$$

where $u_{\tau}=\tilde{u}_{\tau}-\phi_{1} z_{1}\left(\omega_{1}\right), v_{\tau}=\tilde{v}_{\tau}-\phi_{2} z_{2}\left(\omega_{2}\right)$. Both cocycles $\Phi$ and $\tilde{\Phi}$ are equivalent and so we will discuss only the cocycle $\Phi$ induced by Equations (21)-(24) in this paper. Let $B=\{B(\tau, \omega): \tau \in \mathbf{R}, \omega \in \Omega\}$ be a family of bounded nonempty subsets of $H$. Such a family $B$ is called tempered if for every $c>0, \tau \in \mathbf{R}$ and $\omega \in \Omega$,

$$
\lim _{t \rightarrow+\infty} \mathrm{e}^{-c t}\left\|B\left(\tau-t, \theta_{-t} \omega\right)\right\|_{H}^{2}=0,
$$

where the norm $\|B\|$ of set $B$ in $H$ is given by $\|B\|=\sup _{u \in B}\|u\|$. From now on, we will use $\mathcal{D}$ to denote the collection of all tempered families of bounded nonempty subsets of $H$ :

$$
\mathcal{D}=\{B=\{B(\tau, \omega): \tau \in \mathbf{R}, \omega \in \Omega\}: B \text { is tempered in } H\} .
$$

The functions $g$ and $h$ in (1)-(2) are satisfy, for every $\tau \in \mathbf{R}$,

$$
\int_{-\infty}^{0} \mathrm{e}^{\delta s}\left(\|g(s+\tau, \cdot)\|^{2}+\|h(s+\tau, \cdot)\|^{2}\right) \mathrm{d} s<\infty,
$$




$$
\int_{-\infty}^{0} \mathrm{e}^{\delta s}\left\|(-\Delta)^{\frac{s}{2}} h(s+\tau, \cdot)\right\|^{2} \mathrm{~d} s<\infty .
$$

and for every $c>0$,

$$
\lim _{r \rightarrow+\infty} \mathrm{e}^{-c r} \int_{-\infty}^{0} \mathrm{e}^{\delta s}\left(\|g(s-r, \cdot)\|^{2}+\|h(s-r, \cdot)\|^{2}\right) \mathrm{d} s=0 .
$$

Throughout this paper, $C$ denotes a constant which may be different from the context.

\section{Existence of Random Attractor}

This section is devoted to uniform estimates of solutions for the problem (21)-(24), which are useful for constructing random pullback absorbing sets. We begin with the uniform estimates of solutions in $H$.

Lemma 3.1. Under conditions (5)-(8) and (28), for every $\sigma \in \mathbf{R}, \tau \in \mathbf{R}, \omega \in \Omega$ and $B=\{B(\tau, \omega): \tau \in \mathbf{R}, \omega \in \Omega\} \in \mathcal{D}$, there exists $T=T(\tau, \omega, B, \sigma)>0$ such that for all $t \geq T$, the solution $(u, v)$ of problem(21)-(24) satisfies

$$
\begin{aligned}
& \alpha_{2}\left\|u\left(\sigma, \tau-t, \theta_{-\tau} \omega, u_{\tau-t}\right)\right\|^{2}+\alpha_{1}\left\|v\left(\sigma, \tau-t, \theta_{-\tau} \omega, v_{\tau-t}\right)\right\|^{2} \\
& +\frac{\delta}{2} \alpha_{1} \int_{-t}^{\sigma-\tau} \mathrm{e}^{\delta(s+\tau-\sigma)}\left\|v\left(s+\tau, \tau-t, \theta_{-\tau} \omega, v_{\tau-t}\right)\right\|^{2} \mathrm{~d} s \\
& +C \int_{-t}^{\sigma-\tau} \mathrm{e}^{\delta(s+\tau-\sigma)}\left\|u\left(s+\tau, \tau-t, \theta_{-\tau} \omega, u_{\tau-t}\right)\right\|_{H^{s}(\mathbf{U})}^{2} \mathrm{~d} s \\
& +\alpha_{2} \beta_{1} \int_{-t}^{\sigma-\tau} \mathrm{e}^{\delta(s+\tau-\sigma)} \int_{\mathbf{U}}\left|\tilde{u}\left(s+\tau, \tau-t, \theta_{-\tau} \omega, \tilde{u}_{\tau-t}\right)\right|^{p} \mathrm{~d} x \mathrm{~d} s \\
& \leq C+C \int_{-\infty}^{\sigma-\tau} \mathrm{e}^{\delta(s+\tau-\sigma)}\left(\|g(s+\tau)\|^{2}+\|h(s+\tau)\|^{2}+1\right) \mathrm{d} s .
\end{aligned}
$$

where $\left(u_{\tau-t}, v_{\tau-t}\right) \in B\left(\tau-t, \theta_{-t} \omega\right)$.

Proof. It follows from (21) and (22) that

$$
\begin{aligned}
& \frac{\mathrm{d}}{\mathrm{d} t}\left(\frac{\alpha_{2}}{2}\|u\|^{2}+\frac{\alpha_{1}}{2}\|v\|^{2}\right)+\lambda_{1} \alpha_{2}\|u\|^{2}+\lambda_{2} \alpha_{1}\|v\|^{2}+\alpha_{2}\left\|(-\Delta)^{\frac{s}{2}} u\right\|^{2} \\
& =\alpha_{2} \int_{\mathbf{U}} f(x, \tilde{u}) u \mathrm{~d} x+\alpha_{2} \int_{\mathbf{U}} g(t, x) u \mathrm{~d} x+\alpha_{1} \int_{\mathbf{U}} h(t, x) v \mathrm{~d} x \\
& \quad-\alpha_{2}\left((-\Delta)^{s} \phi_{1} z_{1}\left(\theta_{t} \omega_{1}\right), u\right)-\alpha_{1} \alpha_{2}\left(\phi_{2} z_{2}\left(\theta_{t} \omega_{2}\right), u\right)+\alpha_{1} \alpha_{2}\left(\phi_{1} z_{1}\left(\theta_{t} \omega_{1}\right), v\right) .
\end{aligned}
$$

We now estimate each term on the right-hand side of (31). For the first term, by (5) and (6) we obtain

$$
\begin{aligned}
\alpha_{2} \int_{\mathbf{U}} f(x, \tilde{u}) u \mathrm{~d} x= & \alpha_{2} \int_{\mathbf{U}} f(x, \tilde{u})\left(\tilde{u}-\phi_{1} z_{1}\left(\theta_{t} \omega_{1}\right)\right) \mathrm{d} x \\
\leq & -\alpha_{2} \beta_{1} \int_{\mathbf{U}}\left|u+\phi_{1} z_{1}\left(\theta_{t} \omega_{1}\right)\right|^{p} \mathrm{~d} x+\alpha_{2} \int_{\mathbf{U}} \psi_{1}(x) \mathrm{d} x \\
& +\alpha_{2} \beta_{2} \int_{\mathbf{U}}\left|u+\phi_{1} z_{1}\left(\theta_{t} \omega_{1}\right)\right|^{p-1}\left|\phi_{1} z_{1}\left(\theta_{t} \omega_{1}\right)\right| \mathrm{d} x \\
& +\alpha_{2} \int_{\mathbf{U}}\left|\psi_{2}(x)\right|\left|\phi_{1} z_{1}\left(\theta_{t} \omega_{1}\right)\right| \mathrm{d} x \\
\leq & \frac{-\alpha_{2} \beta_{1}}{2} \int_{\mathbf{U}}\left|u+\phi_{1} z_{1}\left(\theta_{t} \omega_{1}\right)\right|^{p} \mathrm{~d} x+C\left(\int_{\mathbf{U}} \psi_{1}(x) \mathrm{d} x+\int_{\mathbf{U}}\left|\psi_{2}(x)\right|^{q} \mathrm{~d} x\right. \\
& \left.+\left|z_{1}\left(\theta_{t} \omega_{1}\right)\right|^{p} \int_{\mathbf{U}}\left|\phi_{1}(x)\right|^{p} \mathrm{~d} x\right) \\
\leq & \frac{-\alpha_{2} \beta_{1}}{2} \int_{\mathbf{U}}\left|u+\phi_{1} z_{1}\left(\theta_{t} \omega_{1}\right)\right|^{p} \mathrm{~d} x+C\left(1+\left|z_{1}\left(\theta_{t} \omega_{1}\right)\right|^{p}\right),
\end{aligned}
$$


where $\psi_{1}, \psi_{2}$ satisfies the assumed conditions.

By the Young inequality, we have the following estimates on the remaining terms on the right-hand side of (31)

$$
\begin{gathered}
\alpha_{2} \int_{\mathbf{U}} g(t, x) u \mathrm{~d} x \leq \frac{\lambda_{1} \alpha_{2}}{8}\|u\|^{2}+\frac{2 \alpha_{2}}{\lambda_{1}}\|g(t)\|^{2}, \\
\alpha_{1} \int_{\mathbf{U}} h(t, x) v \mathrm{~d} x \leq \frac{\lambda_{2} \alpha_{1}}{8}\|v\|^{2}+\frac{2 \alpha_{1}}{\lambda_{2}}\|h(t)\|^{2}, \\
\alpha_{2}\left|\left((-\Delta)^{s} \phi_{1} z_{1}\left(\theta_{t} \omega_{1}\right), u\right)\right| \leq \frac{\alpha_{2}}{2}\left|z_{1}\left(\theta_{t} \omega_{1}\right)\right|^{2}\left\|(-\Delta)^{\frac{s}{2}} \phi_{1}\right\|^{2}+\frac{\alpha_{2}}{2}\left\|(-\Delta)^{\frac{s}{2}} u\right\|^{2}, \\
\alpha_{1} \alpha_{2}\left|\left(\phi_{2} z_{2}\left(\theta_{t} \omega_{2}\right), u\right)\right| \leq \frac{\lambda_{1} \alpha_{2}}{8}\|u\|^{2}+\frac{2}{\lambda_{1}} \alpha_{1}^{2} \alpha_{2}\left|z_{2}\left(\theta_{t} \omega_{2}\right)\right|^{2}\left\|\phi_{2}\right\|^{2}, \\
\alpha_{1} \alpha_{2}\left|\left(\phi_{1} z_{1}\left(\theta_{t} \omega_{1}\right), v\right)\right| \leq \frac{\lambda_{2} \alpha_{1}}{8}\|v\|^{2}+\frac{2}{\lambda_{2}} \alpha_{1} \alpha_{2}^{2}\left|z_{1}\left(\theta_{t} \omega_{1}\right)\right|^{2}\left\|\phi_{1}\right\|^{2} .
\end{gathered}
$$

Since $\delta=\min \left\{\lambda_{1}, \lambda_{2}\right\}$, it follows from (31)-(37) that

$$
\begin{aligned}
& \frac{\mathrm{d}}{\mathrm{d} t}\left(\alpha_{2}\|u\|^{2}+\alpha_{1}\|v\|^{2}\right)+\delta\left(\alpha_{2}\|u\|^{2}+\alpha_{1}\|v\|^{2}\right)+\frac{\delta}{2}\left(\alpha_{2}\|u\|^{2}+\alpha_{1}\|v\|^{2}\right) \\
& +\alpha_{2}\left\|(-\Delta)^{\frac{s}{2}} u\right\|^{2}+\alpha_{2} \beta_{1} \int_{\mathbf{U}}|\tilde{u}|^{p} \mathrm{~d} x \\
& \leq \frac{4 \alpha_{2}}{\lambda_{1}}\|g(t)\|^{2}+\frac{4 \alpha_{1}}{\lambda_{2}}\|h(t)\|^{2}+C\left(1+\left|z_{1}\left(\theta_{t} \omega_{1}\right)\right|^{2}+\left|z_{1}\left(\theta_{t} \omega_{1}\right)\right|^{p}+\left|z_{2}\left(\theta_{t} \omega_{2}\right)\right|^{2}\right) .
\end{aligned}
$$

Multiplying (38) by $\mathrm{e}^{\delta t}$ and then integrating the above inequality on $(\tau-t, \sigma)$ with $\sigma>\tau-t$, we get

$$
\begin{aligned}
& \alpha_{2}\left\|u\left(\sigma, \tau-t, \omega, u_{\tau-t}\right)\right\|^{2}+\alpha_{1}\left\|v\left(\sigma, \tau-t, \omega, v_{\tau-t}\right)\right\|^{2} \\
& +\frac{\delta}{2} \int_{\tau-t}^{\sigma} \mathrm{e}^{\delta(s-\sigma)}\left(\alpha_{2}\left\|u\left(s, \tau-t, \omega, u_{\tau-t}\right)\right\|^{2}+\alpha_{1}\left\|v\left(s, \tau-t, \omega, v_{\tau-t}\right)\right\|^{2}\right) \mathrm{d} s \\
& +\alpha_{2} \int_{\tau-t}^{\sigma} \mathrm{e}^{\delta(s-\sigma)}\left\|(-\Delta)^{\frac{s}{2}} u\left(s, \tau-t, \omega, u_{\tau-t}\right)\right\|^{2} \mathrm{~d} s \\
& +\alpha_{2} \beta_{1} \int_{\tau-t}^{\sigma} \mathrm{e}^{\delta(s-\sigma)} \int_{\mathbf{U}}\left|\tilde{u}\left(s, \tau-t, \omega, \tilde{u}_{\tau-t}\right)\right|^{p} \mathrm{~d} x \mathrm{~d} s \\
& \leq \mathrm{e}^{\delta(\tau-t-\sigma)}\left(\alpha_{2}\left\|u_{\tau-t}\right\|^{2}+\alpha_{1}\left\|v_{\tau-t}\right\|^{2}\right) \\
& +\frac{4 \alpha_{2}}{\lambda_{1}} \int_{\tau-t}^{\sigma} \mathrm{e}^{\delta(s-\sigma)}\|g(s)\|^{2} \mathrm{~d} s+\frac{4 \alpha_{1}}{\lambda_{2}} \int_{\tau-t}^{\sigma} \mathrm{e}^{\delta(s-\sigma)}\|h(s)\|^{2} \mathrm{~d} s \\
& +C \int_{\tau-t}^{\sigma} \mathrm{e}^{\delta(s-\sigma)}\left(1+\left|z_{1}\left(\theta_{s} \omega_{1}\right)\right|^{2}+\left|z_{1}\left(\theta_{s} \omega_{1}\right)\right|^{p}+\left|z_{2}\left(\theta_{s} \omega_{2}\right)\right|^{2}\right) \mathrm{d} s .
\end{aligned}
$$

Replacing $\omega$ by $\theta_{-\tau} \omega$ in the above, after changes of variables, we obtain

$$
\begin{aligned}
& \alpha_{2}\left\|u\left(\sigma, \tau-t, \theta_{-\tau} \omega, u_{\tau-t}\right)\right\|^{2}+\alpha_{1}\left\|v\left(\sigma, \tau-t, \theta_{-\tau} \omega, v_{\tau-t}\right)\right\|^{2} \\
& +\frac{\delta}{2} \alpha_{1} \int_{-t}^{\sigma-\tau} \mathrm{e}^{\delta(s+\tau-\sigma)}\left\|v\left(s+\tau, \tau-t, \theta_{-\tau} \omega, v_{\tau-t}\right)\right\|^{2} \mathrm{~d} s \\
& +\frac{\delta}{2} \alpha_{2} \int_{-t}^{\sigma-\tau} \mathrm{e}^{\delta(s+\tau-\sigma)}\left\|u\left(s+\tau, \tau-t, \theta_{-\tau} \omega, u_{\tau-t}\right)\right\|^{2} \mathrm{~d} s \\
& +\frac{\alpha_{2}}{2} C(n, s) \int_{-t}^{\sigma-\tau} \mathrm{e}^{\delta(s+\tau-\sigma)}\left\|u\left(s+\tau, \tau-t, \theta_{-\tau} \omega, u_{\tau-t}\right)\right\|_{\dot{H}^{s}(\mathbf{U})}^{2} \mathrm{~d} s \\
& +\alpha_{2} \beta_{1} \int_{-t}^{\sigma-\tau} \mathrm{e}^{\delta(s+\tau-\sigma)} \int_{\mathbf{U}}\left|\tilde{u}\left(s+\tau, \tau-t, \theta_{-\tau} \omega, \tilde{u}_{\tau-t}\right)\right|^{p} \mathrm{~d} x \mathrm{~d} s
\end{aligned}
$$




$$
\begin{aligned}
\leq & \mathrm{e}^{\delta(\tau-t-\sigma)}\left(\left.\alpha_{2}\left\|u_{\tau-t}\right\|\right|^{2}+\alpha_{1}\left\|v_{\tau-t}\right\|^{2}\right)+\frac{4 \alpha_{2}}{\lambda_{1}} \int_{-t}^{\sigma-\tau} \mathrm{e}^{\delta(s+\tau-\sigma)}\|g(s+\tau)\|^{2} \mathrm{~d} s \\
& +\frac{4 \alpha_{1}}{\lambda_{2}} \int_{-t}^{\sigma-\tau} \mathrm{e}^{\delta(s+\tau-\sigma)}\|h(s+\tau)\|^{2} \mathrm{~d} s \\
& +C \int_{-t}^{\sigma-\tau} \mathrm{e}^{\delta(s+\tau-\sigma)}\left(1+\left|z_{1}\left(\theta_{s} \omega_{1}\right)\right|^{2}+\left|z_{1}\left(\theta_{s} \omega_{1}\right)\right|^{p}+\left|z_{2}\left(\theta_{s} \omega_{2}\right)\right|^{2}\right) \mathrm{d} s .
\end{aligned}
$$

We now estimates the first term on the right-hand side of (40). Due to $\left(u_{\tau-t}, v_{\tau-t}\right) \in B\left(\tau-t, \theta_{-t} \omega\right)$ and $B=\{B(\tau, \omega): \tau \in \mathbf{R}, \omega \in \Omega\}$ is tempered, we find

$$
\begin{aligned}
& \mathrm{e}^{\delta(\tau-t-\sigma)}\left(\alpha_{2}\left\|u_{\tau-t}\right\|^{2}+\alpha_{1}\left\|v_{\tau-t}\right\|^{2}\right) \\
& =\mathrm{e}^{\delta(\tau-\sigma)} \mathrm{e}^{-\delta t}\left(\alpha_{2}\left\|u_{\tau-t}\right\|^{2}+\alpha_{1}\left\|v_{\tau-t}\right\|^{2}\right) \\
& \leq \mathrm{e}^{\delta(\tau-\sigma)} \mathrm{e}^{-\delta t}\left(\alpha_{1}+\alpha_{2}\right)\left\|B\left(\tau-t, \theta_{-t} \omega\right)\right\|^{2} \rightarrow 0 \text { as } t \rightarrow+\infty .
\end{aligned}
$$

Therefore, there exists $T=T(\tau, \omega, B, \sigma)>0$ such that for all $t \geq T$

$$
\mathrm{e}^{\delta(\tau-t-\sigma)}\left(\alpha_{2}\left\|u_{\tau-t}\right\|^{2}+\alpha_{1}\left\|v_{\tau-t}\right\|^{2}\right) \leq 1 .
$$

For the remaining terms on the right-hand side of (40), we have

$$
\begin{gathered}
\frac{4 \alpha_{2}}{\lambda_{1}} \int_{-t}^{\sigma-\tau} \mathrm{e}^{\delta(s+\tau-\sigma)}\|g(s+\tau)\|^{2} \mathrm{~d} s \leq \frac{4 \alpha_{2}}{\lambda_{1}} \int_{-\infty}^{\sigma-\tau} \mathrm{e}^{\delta(s+\tau-\sigma)}\|g(s+\tau)\|^{2} \mathrm{~d} s, \\
\frac{4 \alpha_{1}}{\lambda_{2}} \int_{-t}^{\sigma-\tau} \mathrm{e}^{\delta(s+\tau-\sigma)}\|h(s+\tau)\|^{2} \mathrm{~d} s \leq \frac{4 \alpha_{1}}{\lambda_{2}} \int_{-\infty}^{\sigma-\tau} \mathrm{e}^{\delta(s+\tau-\sigma)}\|h(s+\tau)\|^{2} \mathrm{~d} s, \\
C \int_{-t}^{\sigma-\tau} \mathrm{e}^{\delta(s+\tau-\sigma)}\left(1+\left|z_{1}\left(\theta_{s} \omega_{1}\right)\right|^{2}+\left|z_{1}\left(\theta_{s} \omega_{1}\right)\right|^{p}+\left|z_{2}\left(\theta_{s} \omega_{2}\right)\right|^{2}\right) \mathrm{d} s \\
\leq C \int_{-\infty}^{\sigma-\tau} \mathrm{e}^{\delta(s+\tau-\sigma)}\left(1+\left|z_{1}\left(\theta_{s} \omega_{1}\right)\right|^{2}+\left|z_{1}\left(\theta_{s} \omega_{1}\right)\right|^{p}+\left|z_{2}\left(\theta_{s} \omega_{2}\right)\right|^{2}\right) \mathrm{d} s .
\end{gathered}
$$

By (40)-(45) and (17) we obtain, for all $t \geq T$,

$$
\begin{aligned}
& \alpha_{2}\left\|u\left(\sigma, \tau-t, \theta_{-\tau} \omega, u_{\tau-t}\right)\right\|^{2}+\alpha_{1}\left\|v\left(\sigma, \tau-t, \theta_{-\tau} \omega, v_{\tau-t}\right)\right\|^{2} \\
& +\frac{\delta}{2} \int_{-t}^{\sigma-\tau} \mathrm{e}^{\delta(s+\tau-\sigma)} \alpha_{1}\left\|v\left(s+\tau, \tau-t, \theta_{-\tau} \omega, v_{\tau-t}\right)\right\|^{2} \mathrm{~d} s \\
& +C \int_{-t}^{\sigma-\tau} \mathrm{e}^{\delta(s+\tau-\sigma)}\left\|u\left(s+\tau, \tau-t, \theta_{-\tau} \omega, u_{\tau-t}\right)\right\|_{H^{s}(\mathbf{U})}^{2} \mathrm{~d} s \\
& +\alpha_{2} \beta_{1} \int_{-t}^{\sigma-\tau} \mathrm{e}^{\delta(s+\tau-\sigma)} \int_{\mathbf{U}}\left|\tilde{u}\left(s+\tau, \tau-t, \theta_{-\tau} \omega, \tilde{u}_{\tau-t}\right)\right|^{p} \mathrm{~d} x \mathrm{~d} s \\
& \leq C+C \int_{-\infty}^{\sigma-\tau} \mathrm{e}^{\delta(s+\tau-\sigma)}\left(\|g(s+\tau)\|^{2}+\|h(s+\tau)\|^{2}+1\right) \mathrm{d} s .
\end{aligned}
$$

From (28), the desired estimates follow immediately.

We now derive uniform estimates of $u$ in $H^{s}(U)$.

Lemma 3.2. Under conditions (5)-(8) and (28), for every $\sigma \in \mathbf{R}, \tau \in \mathbf{R}, \omega \in \Omega$ and $B=\{B(\tau, \omega): \tau \in \mathbf{R}, \omega \in \Omega\} \in \mathcal{D}$, there exists $T(\tau, \omega, B, \sigma) \geq 2$ such that for all $t \geq T$ and $\sigma \in[\tau-1, \tau]$, the solution $u$ of problem (21) with $\left(u_{\tau-t}, v_{\tau-t}\right) \in B\left(\tau-t, \theta_{-t} \omega\right)$ satisfies

$$
\begin{aligned}
& \left\|u\left(\sigma, \tau-t, \theta_{-\tau} \omega, u_{\tau-t}\right)\right\|_{\dot{H}^{s}(\mathbf{U})}^{2} \\
& \leq C+C \int_{-\infty}^{\sigma-\tau} \mathrm{e}^{\delta(s+\tau-\sigma)}\left(\|g(s+\tau)\|^{2}+\|h(s+\tau)\|^{2}+1\right) \mathrm{d} s .
\end{aligned}
$$


Proof. Multiplying (21) by $(-\Delta)^{s} u$, we obtain

$$
\begin{aligned}
& \frac{1}{2} \frac{\mathrm{d}}{\mathrm{d} t}\left\|(-\Delta)^{\frac{s}{2}} u\right\|^{2}+\left\|(-\Delta)^{s} u\right\|^{2}+\lambda_{1} \int_{\mathbf{U}} u(-\Delta)^{s} u \mathrm{~d} x+\alpha_{1} \int_{\mathbf{U}} v(-\Delta)^{s} u \mathrm{~d} x \\
= & \int_{\mathbf{U}} f(x, \tilde{u})(-\Delta)^{s} u \mathrm{~d} x+\int_{\mathbf{U}} g(t, x)(-\Delta)^{s} u \mathrm{~d} x \\
& -\int_{\mathbf{U}}(-\Delta)^{s} \phi_{1} z_{1}\left(\theta_{t} \omega_{1}\right)(-\Delta)^{s} u \mathrm{~d} x-\alpha_{1} \int_{\mathbf{U}} \phi_{2} z_{2}\left(\theta_{t} \omega_{2}\right)(-\Delta)^{s} u \mathrm{~d} x .
\end{aligned}
$$

For the nonlinear term in (47), by (6)-(8) we have

$$
\begin{aligned}
& 2 \int_{\mathrm{U}} f(x, \tilde{u})(-\Delta)^{s} u \mathrm{~d} x \\
& =2 \int_{\mathbf{U}} f(x, \tilde{u})(-\Delta)^{s} \tilde{u} \mathrm{~d} x-2 \int_{\mathbf{U}} f(x, \tilde{u})(-\Delta)^{s} \phi_{1} z_{1}\left(\theta_{t} \omega_{1}\right) \mathrm{d} x \\
& \leq C(n, s) \int_{\mathbf{U}} \int_{\mathbf{U}} \frac{(f(x, \tilde{u}(x))-f(y, \tilde{u}(y)))(\tilde{u}(x)-\tilde{u}(y))}{|x-y|^{n+2 s}} \mathrm{~d} x \mathrm{~d} y \\
& +2\left|z_{1}\left(\theta_{t} \omega_{1}\right)\right| \int_{\mathbf{U}}|f(x, \tilde{u})|(-\Delta)^{s} \phi_{1} \mid \mathrm{d} x \\
& \leq C(n, s) \int_{\mathbf{U}} \int_{\mathbf{U}} \frac{(f(x, \tilde{u}(x))-f(y, \tilde{u}(x)))(\tilde{u}(x)-\tilde{u}(y))}{|x-y|^{n+2 s}} \mathrm{~d} x \mathrm{~d} y \\
& +C(n, s) \int_{\mathrm{U}} \int_{\mathrm{U}} \frac{(f(y, \tilde{u}(x))-f(y, \tilde{u}(y)))(\tilde{u}(x)-\tilde{u}(y))}{|x-y|^{n+2 s}} \mathrm{~d} x \mathrm{~d} y \\
& +2\left|z_{1}\left(\theta_{t} \omega_{1}\right)\right| \int_{\mathbf{U}}|f(x, \tilde{u})|\left|(-\Delta)^{s} \phi_{1}\right| \mathrm{d} x \\
& \leq C(n, s) \int_{\mathrm{U}} \int_{\mathrm{U}} \frac{\left|\psi_{4}(x)-\psi_{4}(y)\right||\tilde{u}(x)-\tilde{u}(y)|}{|x-y|^{n+2 s}} \mathrm{~d} x \mathrm{~d} y \\
& +C(n, s) \int_{\mathrm{U}} \int_{\mathrm{U}} \frac{\left|\psi_{3}(y)\right||\tilde{u}(x)-\tilde{u}(y)|^{2}}{|x-y|^{n+2 s}} \mathrm{~d} x \mathrm{~d} y \\
& +2\left|z_{1}\left(\theta_{t} \omega_{1}\right)\right| \int_{\mathbf{U}}|f(x, \tilde{u})|\left|(-\Delta)^{s} \phi_{1}\right| \mathrm{d} x \\
& \leq \frac{1}{2} C(n, s) \int_{\mathbf{U}} \int_{\mathbf{U}} \frac{\left|\psi_{4}(x)-\psi_{4}(y)\right|^{2}}{|x-y|^{n+2 s}} \mathrm{~d} x \mathrm{~d} y \\
& +\frac{1}{2} C(n, s) \int_{\mathbf{U}} \int_{\mathbf{U}} \frac{|\tilde{u}(x)-\tilde{u}(y)|^{2}}{|x-y|^{n+2 s}} \mathrm{~d} x \mathrm{~d} y \\
& +C(n, s) \int_{\mathbf{U}}\left|\psi_{3}(y)\right| \int_{\mathbf{U}} \frac{|\tilde{u}(x)-\tilde{u}(y)|^{2}}{|x-y|^{n+2 s}} \mathrm{~d} x \mathrm{~d} y \\
& +2\left|z_{1}\left(\theta_{t} \omega_{1}\right)\right| \int_{\mathbf{U}} \beta_{2}|\tilde{u}|^{p-1}\left|(-\Delta)^{s} \phi_{1}\right| \mathrm{d} x \\
& +2\left|z_{1}\left(\theta_{t} \omega_{1}\right)\right| \int_{\mathbf{U}} \psi_{2}(x)\left|(-\Delta)^{s} \phi_{1}\right| \mathrm{d} x \\
& \leq\left\|\psi_{4}\right\|_{H^{s}(\mathbf{U})}^{2}+\left\|(-\Delta)^{\frac{s}{2}} \tilde{u}\right\|^{2}+2\left\|\psi_{3}\right\|_{L^{\infty}(\mathbf{U})}\left\|(-\Delta)^{\frac{s}{2}} \tilde{u}\right\|^{2} \\
& +2 \beta_{2}\left|z_{1}\left(\theta_{t} \omega_{1}\right)\right| \int_{\mathrm{U}}|\tilde{u}|^{p-1}\left|(-\Delta)^{s} \phi_{1}\right| \mathrm{d} x \\
& +2\left|z_{1}\left(\theta_{t} \omega_{1}\right)\right| \int_{\mathbf{U}} \psi_{2}(x)\left|(-\Delta)^{s} \phi_{1}\right| \mathrm{d} x \text {. }
\end{aligned}
$$


We now estimate the remaining terms on the right-hand side of (47). Using the Hölder inequality and Young inequality, we can get

$$
\begin{gathered}
\int_{\mathbf{U}} g(t, x)(-\Delta)^{s} u \mathrm{~d} x \leq \frac{1}{8}\left\|(-\Delta)^{s} u\right\|^{2}+2\|g(t)\|^{2}, \\
\left|\int_{\mathbf{U}}(-\Delta)^{s} \phi_{1} z_{1}\left(\theta_{t} \omega_{1}\right)(-\Delta)^{s} u \mathrm{~d} x\right| \leq \frac{1}{8}\left\|(-\Delta)^{s} u\right\|^{2}+2\left|z_{1}\left(\theta_{t} \omega_{1}\right)\right|^{2}\left\|(-\Delta)^{s} \phi_{1}\right\|^{2}, \\
\left|\alpha_{1} \int_{\mathbf{U}} \phi_{2} z_{2}\left(\theta_{t} \omega_{2}\right)(-\Delta)^{s} u \mathrm{~d} x\right| \leq \frac{1}{8}\left\|(-\Delta)^{s} u\right\|^{2}+2 \alpha_{1}^{2}\left|z_{2}\left(\theta_{t} \omega_{2}\right)\right|^{2}\left\|\phi_{2}\right\|^{2} .
\end{gathered}
$$

For the last term on the left-hand side of (47), we obtain

$$
\left|\alpha_{1} \int_{\mathbf{U}} v(-\Delta)^{s} u \mathrm{~d} x\right| \leq \frac{1}{8}\left\|(-\Delta)^{s} u\right\|^{2}+2 \alpha_{1}^{2}\|v\|^{2} .
$$

It follows from (47)-(52) that

$$
\begin{aligned}
& \frac{\mathrm{d}}{\mathrm{d} t}\left\|(-\Delta)^{\frac{s}{2}} u\right\|^{2}+2 \lambda_{1}\left\|(-\Delta)^{\frac{s}{2}} u\right\|^{2}+\left\|(-\Delta)^{s} u\right\|^{2} \\
& \leq C\left(\left\|(-\Delta)^{\frac{s}{2}} \tilde{u}\right\|^{2}+\|\tilde{u}\|_{L^{p}(\mathbf{U})}^{p}+\|v\|^{2}+\left|z_{1}\left(\theta_{t} \omega_{1}\right)\right|^{p}\right. \\
& \left.\quad+\left|z_{1}\left(\theta_{t} \omega_{1}\right)\right|^{2}+\left|z_{2}\left(\theta_{t} \omega_{2}\right)\right|^{2}+\|g(t)\|^{2}+1\right) .
\end{aligned}
$$

Given $t \in \mathbf{R}^{+}, \tau \in \mathbf{R}$ and $\omega \in \Omega$, let $\sigma \in(\tau-1, \tau)$ and $r \in(\tau-2, \tau-1)$. Multiplying (53) by $\mathrm{e}^{\delta t}$, first integrating over $(r, \sigma)$ and then integrating with respect to $r$ on $(\tau-2, \tau-1)$, replacing $\omega$ by $\theta_{-\tau} \omega$ we have

$$
\begin{aligned}
& \left\|(-\Delta)^{\frac{s}{2}} u\left(\sigma, \tau-t, \theta_{-\tau} \omega, u_{\tau-t}\right)\right\| \\
& \leq\left.\int_{\tau-2}^{\tau-1} \mathrm{e}^{\delta(r-\sigma)}\left\|(-\Delta)^{\frac{s}{2}} u\left(r, \tau-t, \theta_{-\tau} \omega, u_{\tau-t}\right)\right\|\right|^{2} \mathrm{~d} r \\
& +C \int_{\tau-2}^{\tau-1} \int_{r}^{\sigma} \mathrm{e}^{\delta(\xi-\sigma)}\left(\left\|(-\Delta)^{\frac{s}{2}} \tilde{u}\left(\xi, \tau-t, \theta_{-\tau} \omega, \tilde{u}_{\tau-t}\right)\right\|^{2}\right. \\
& +\left\|\tilde{u}\left(\xi, \tau-t, \theta_{-\tau} \omega, \tilde{u}_{\tau-t}\right)\right\|_{L^{p}(\mathbf{U})}^{p}+\left\|v\left(\xi, \tau-t, \theta_{-\tau} \omega, v_{\tau-t}\right)\right\|^{2} \\
& \left.+\left|z_{1}\left(\theta_{\xi-\tau} \omega_{1}\right)\right|^{p}+\left|z_{1}\left(\theta_{\xi-\tau} \omega_{1}\right)\right|^{2}+\left|z_{2}\left(\theta_{\xi-\tau} \omega_{2}\right)\right|^{2}+\|g(\xi)\|^{2}+1\right) \mathrm{d} \xi \mathrm{d} r \\
& \leq \int_{-2}^{-1} \mathrm{e}^{\delta(r+\tau-\sigma)}\left\|(-\Delta)^{\frac{s}{2}} u\left(r+\tau, \tau-t, \theta_{-\tau} \omega, u_{\tau-t}\right)\right\|^{2} \mathrm{~d} r \\
& +C \int_{-2}^{\sigma-\tau} \mathrm{e}^{\delta(\xi+\tau-\sigma)}\left(\left\|(-\Delta)^{\frac{s}{2}} \tilde{u}\left(\xi+\tau, \tau-t, \theta_{-\tau} \omega, \tilde{u}_{\tau-t}\right)\right\|^{2}\right. \\
& +\left\|\tilde{u}\left(\xi+\tau, \tau-t, \theta_{-\tau} \omega, \tilde{u}_{\tau-t}\right)\right\|_{L^{p}(\mathbf{U})}^{p}+\left\|v\left(\xi+\tau, \tau-t, \theta_{-\tau} \omega, v_{\tau-t}\right)\right\|^{2} \\
& \left.+\left|z_{1}\left(\theta_{\xi} \omega_{1}\right)\right|^{p}+\left|z_{1}\left(\theta_{\xi} \omega_{1}\right)\right|^{2}+\left|z_{2}\left(\theta_{\xi} \omega_{2}\right)\right|^{2}+\|g(\xi+\tau)\|^{2}+1\right) \mathrm{d} \xi .
\end{aligned}
$$

Let $T$ be the constant in Lemma 4.1 and $T_{0}=\max \{2, T\}$. By the fact that 


$$
\begin{aligned}
\tilde{u} & =u+\phi_{1} z_{1}\left(\theta_{t} \omega_{1}\right), \text { from } \sigma \in(\tau-1, \tau) \text { and Lemma 4.1, for all } t \geq T_{0} \text {, we obtain } \\
\|(-\Delta)^{\frac{s}{2}} u\left(\sigma, \tau-t, \theta_{-\tau} \omega, u_{\tau-t}\right) & \|^{2} \\
\leq & C\left(\int_{-2}^{\sigma-\tau} \mathrm{e}^{\delta(\xi+\tau-\sigma)}\left\|(-\Delta)^{\frac{s}{2}} u\left(\xi+\tau, \tau-t, \theta_{-\tau} \omega, u_{\tau-t}\right)\right\| \|^{2} \mathrm{~d} \xi\right. \\
& +\int_{-2}^{\sigma-\tau} \mathrm{e}^{\delta(\xi+\tau-\sigma)}\left\|\tilde{u}\left(\xi+\tau, \tau-t, \theta_{-\tau} \omega, \tilde{u}_{\tau-t}\right)\right\|_{L^{p}(\mathbf{U})}^{p} \mathrm{~d} \xi \\
& +\int_{-2}^{\sigma-\tau} \mathrm{e}^{\delta(\xi+\tau-\sigma)}\left\|v\left(\xi+\tau, \tau-t, \theta_{-\tau} \omega, v_{\tau-t}\right)\right\|^{2} \mathrm{~d} \xi \\
& \left.+\int_{-2}^{\sigma-\tau} \mathrm{e}^{\delta(\xi+\tau-\sigma)}\left(\left|z_{1}\left(\theta_{\xi} \omega_{1}\right)\right|^{p}+\left|z_{1}\left(\theta_{\xi} \omega_{1}\right)\right|^{2}+\left|z_{2}\left(\theta_{\xi} \omega_{2}\right)\right|^{2}+\|g(\xi+\tau)\|^{2}+1\right) \mathrm{d} \xi\right) .
\end{aligned}
$$

From (17)-(18) and Lemma 4.1, we immediately concludes the proof. $\square$

Note that Equation (22) has no any smoothing effect on the solutions. To overcome this difficulty, we must decompose the solution operator into two parts. Let $v_{1}$ and $v_{2}$ be the solution of the following problems, respectively,

$$
\begin{gathered}
\frac{\mathrm{d} v_{1}}{\mathrm{~d} t}+\lambda_{2} v_{1}=0, \\
v_{1}(\tau-t)=v(\tau-t, x), \\
\frac{\mathrm{d} v_{2}}{\mathrm{~d} t}+\lambda_{2} v_{2}=\alpha_{2} u+h(t, x)+\alpha_{2} \phi_{1} z_{1}\left(\theta_{t} \omega_{1}\right), \\
v_{2}(\tau-t)=0 .
\end{gathered}
$$

Then $v=v_{1}+v_{2}$. Multiplying (55) by $v_{1}$, we obtain

$$
\left\|v_{1}\left(\tau, \tau-t, \theta_{-\tau} \omega, v_{1, \tau-t}\right)\right\|^{2}=\mathrm{e}^{-2 \lambda_{2} t}\left\|v_{1, \tau-t}\right\|^{2} .
$$

Lemma 3.3. Under conditions (5)-(7) and (29), for every $\tau \in \mathbf{R}, \omega \in \Omega$ and $B=\{B(\tau, \omega): \tau \in \mathbf{R}, \omega \in \Omega\} \in \mathcal{D}$, there exists $T(\tau, \omega, B)$ such that for all $t \geq T$, the solution $v_{2}$ of problem (57)-(58) satisfies

$$
\begin{aligned}
& \left\|(-\Delta)^{\frac{s}{2}} v_{2}\left(\tau, \tau-t, \theta_{-\tau} \omega, 0\right)\right\|^{2} \\
& \leq C+C \int_{-\infty}^{0} \mathrm{e}^{\delta r}\left(\|g(r+\tau)\|^{2}+\|h(r+\tau)\|^{2}+\left\|(-\Delta)^{\frac{s}{2}} h(r+\tau)\right\|^{2}+1\right) \mathrm{d} r .
\end{aligned}
$$

Proof. Multiplying (57) by $(-\Delta)^{s} v_{2}$, we obtain

$$
\begin{aligned}
& \frac{1}{2} \frac{\mathrm{d}}{\mathrm{d} t}\left\|(-\Delta)^{\frac{s}{2}} v_{2}\right\|^{2}+\lambda_{2}\left\|(-\Delta)^{\frac{s}{2}} v_{2}\right\|^{2} \\
& =\alpha_{2} \int_{\mathbf{U}} u(-\Delta)^{s} v_{2} \mathrm{~d} x+\int_{\mathbf{U}} h(t, x)(-\Delta)^{s} v_{2} \mathrm{~d} x+\alpha_{2} \int_{\mathbf{U}} \phi_{1} z_{1}\left(\theta_{t} \omega_{1}\right)(-\Delta)^{s} v_{2} \mathrm{~d} x .
\end{aligned}
$$

For the first term on the right-hand side of (61), we can get

$$
\begin{aligned}
\alpha_{2} \int_{\mathrm{U}} u(-\Delta)^{s} v_{2} \mathrm{~d} x & \leq \alpha_{2}\left\|(-\Delta)^{\frac{s}{2}} v_{2}\right\|\left\|(-\Delta)^{\frac{s}{2}} u\right\| \\
& \leq \frac{\lambda_{2}}{8}\left\|(-\Delta)^{\frac{s}{2}} v_{2}\right\|^{2}+\frac{2 \alpha_{2}^{2}}{\lambda_{2}}\left\|(-\Delta)^{\frac{s}{2}} u\right\|^{2} .
\end{aligned}
$$


For the second term on the right-hand side of (61), we have

$$
\int_{\mathbf{U}} h(t, x)(-\Delta)^{s} v_{2} \mathrm{~d} x \leq \frac{\lambda_{2}}{4}\left\|(-\Delta)^{\frac{s}{2}} v_{2}\right\|^{2}+\frac{1}{\lambda_{2}}\left\|(-\Delta)^{\frac{s}{2}} h(t)\right\|^{2} .
$$

For the last term, we have

$$
\alpha_{2} \int_{\mathbf{U}} \phi_{1} z_{1}\left(\theta_{t} \omega_{1}\right)(-\Delta)^{s} v_{2} \mathrm{~d} x \leq \frac{\lambda_{2}}{8}\left\|(-\Delta)^{\frac{s}{2}} v_{2}\right\|^{2}+\frac{2 \alpha_{2}^{2}}{\lambda_{2}}\left|z_{1}\left(\theta_{t} \omega_{1}\right)\right|^{2}\left\|(-\Delta)^{\frac{s}{2}} \phi_{1}\right\|^{2} .
$$

It follows from (61)-(64) that

$$
\begin{aligned}
& \frac{\mathrm{d}}{\mathrm{d} t}\left\|(-\Delta)^{\frac{s}{2}} v_{2}\right\|^{2}+\delta\left\|(-\Delta)^{\frac{s}{2}} v_{2}\right\|^{2} \\
& \leq C\left(\left\|(-\Delta)^{\frac{s}{2}} u\right\|^{2}+\left\|(-\Delta)^{\frac{s}{2}} h(t)\right\|^{2}+\left|z_{1}\left(\theta_{t} \omega_{1}\right)\right|^{2}\left\|(-\Delta)^{\frac{s}{2}} \phi_{1}\right\|^{2}\right) .
\end{aligned}
$$

Multiplying (65) by $\mathrm{e}^{\delta t}$, integrating over $(\tau-t, \tau)$, and then replacing $\omega$ by $\theta_{-\tau} \omega$ we have

$$
\begin{aligned}
& \left\|(-\Delta)^{\frac{s}{2}} v_{2}\left(\tau, \tau-t, \theta_{-\tau} \omega, 0\right)\right\|^{2} \\
& \leq C \int_{-t}^{0} \mathrm{e}^{\delta r}\left\|(-\Delta)^{\frac{s}{2}} u\left(r+\tau, \tau-t, \theta_{-\tau} \omega, u_{\tau-t}\right)\right\|^{2} \mathrm{~d} r \\
& \quad+C \int_{-\infty}^{0} \mathrm{e}^{\delta r}\left\|(-\Delta)^{\frac{s}{2}} h(r+\tau)\right\|^{2} \mathrm{~d} r+C\left\|(-\Delta)^{\frac{s}{2}} \phi_{1}\right\|^{2} \int_{-\infty}^{0} \mathrm{e}^{\delta r}\left|z_{1}\left(\theta_{r} \omega_{1}\right)\right|^{2} \mathrm{~d} r,
\end{aligned}
$$

which along with Lemma 4.1 and (17) conclude the proof.

In the following, we will prove the existence of $\mathcal{D}$-pullback random attractor for problem (21)-(24).

Lemma 3.4. Suppose (5)-(7) and (30) hold. Then the continuous cocycle $\Phi$ of problem (21) -(24) has a closed measurable $\mathcal{D}$-pullback absorbing set $K=\{K(\tau, \omega): \tau \in \mathbf{R}, \omega \in \Omega\}$ which is given by

$$
K(\tau, \omega)=\left\{(u, v) \in H:\|u\|^{2}+\|v\|^{2} \leq R(\tau, \omega)\right\},
$$

where $R(\tau, \omega)$ is defined by

$$
R(\tau, \omega)=C+C \int_{-\infty}^{0} \mathrm{e}^{\delta s}\left(\|g(s+\tau)\|^{2}+\|h(s+\tau)\|^{2}+1\right) \mathrm{d} s .
$$

Then for every $\tau \in \mathbf{R}, \omega \in \Omega$ and $B=\{B(\tau, \omega): \tau \in \mathbf{R}, \omega \in \Omega\} \in \mathcal{D}$, there exists $T=T(\tau, \omega, B)>0$ such that the solution $(u, v)$ of problem (21)-(24) with $\left(u_{\tau-t}, v_{\tau-t}\right) \in B\left(\tau-t, \theta_{-t} \omega\right)$ satisfies, for all $t \geq T$,

$$
\left(u\left(\tau, \tau-t, \theta_{-\tau} \omega, u_{\tau-t}\right), v\left(\tau, \tau-t, \theta_{-\tau} \omega, v_{\tau-t}\right)\right) \in K(\tau, \omega) .
$$

In addition, the random variable $R(\tau, \omega)$ as in (68) is tempered, i.e., for any $c>0$,

$$
\lim _{t \rightarrow+\infty} \mathrm{e}^{-c t} R\left(\tau-t, \theta_{-t} \omega\right)=0 .
$$

Proof. As a special case of Lemma 4.1 with $\sigma=\tau$, we obtain (69) immediate- 
ly. Then we have

$$
\Phi\left(t, \tau-t, \theta_{-t} \omega, B\left(\tau-t, \theta_{-t} \omega\right)\right) \subseteq K(\tau, \omega) .
$$

We now verify (70). By (68)

$$
R\left(\tau-t, \theta_{-t} \omega\right)=C+C \int_{-\infty}^{0} \mathrm{e}^{\delta s}\left(\|g(s+\tau-t)\|^{2}+\|h(s+\tau-t)\|^{2}+1\right) \mathrm{d} s .
$$

We have by (30)

$$
\begin{aligned}
& \lim _{t \rightarrow+\infty} \mathrm{e}^{-c t} R\left(\tau-t, \theta_{-t} \omega\right) \\
& \leq \lim _{t \rightarrow+\infty} \mathrm{e}^{-c t}\left(C+C \int_{-\infty}^{0} \mathrm{e}^{\delta s}\left(\|g(s+\tau-t)\|^{2}+\|h(s+\tau-t)\|^{2}+1\right) \mathrm{d} s\right) \\
& \leq \lim _{r \rightarrow+\infty} \mathrm{e}^{-c \tau} \mathrm{e}^{-c r}\left(C+C \int_{-\infty}^{0} \mathrm{e}^{\delta s}\left(\|g(s-r)\|^{2}+\|h(s-r)\|^{2}+1\right) \mathrm{d} s\right) \\
& =0 .
\end{aligned}
$$

Next, we establish the $\mathcal{D}$-pullback asymptotic compactness of $\Phi$ in $H$, for this purpose, we need to split $\Phi$ as follows. Given $t \in \mathbf{R}^{+}, \tau \in \mathbf{R}, \omega \in \Omega$ and $\left(u_{\tau}, v_{\tau}\right) \in H$, Let

$$
\Phi_{1}\left(t, \tau, \omega,\left(u_{\tau}, v_{\tau}\right)\right)=\left(0, v_{1}\left(t+\tau, \tau, \theta_{-\tau} \omega, v_{\tau}\right)\right),
$$

and

$$
\Phi_{2}\left(t, \tau, \omega,\left(u_{\tau}, v_{\tau}\right)\right)=\left(u\left(t+\tau, \tau, \theta_{-\tau} \omega, u_{\tau}\right), v_{2}\left(t+\tau, \tau, \theta_{-\tau} \omega, 0\right)\right) .
$$

where $v_{1}\left(t+\tau, \tau, \theta_{-\tau} \omega, v_{\tau}\right)$ is the solution of (55) with initial condition $v_{\tau}$ at initial time $\tau$, and $v_{2}\left(t+\tau, \tau, \theta_{-\tau} \omega, 0\right)$ is the solution of (57). By (25) we find that for every $t \in \mathbf{R}^{+}, \tau \in \mathbf{R}, \omega \in \Omega$, and $\left(u_{\tau}, v_{\tau}\right) \in H$

$$
\Phi\left(t, \tau, \omega,\left(u_{\tau}, v_{\tau}\right)\right)=\Phi_{1}\left(t, \tau, \omega,\left(u_{\tau}, v_{\tau}\right)\right)+\Phi_{2}\left(t, \tau, \omega,\left(u_{\tau}, v_{\tau}\right)\right) .
$$

The $\mathcal{D}$-pullback asymptotic compactness of $\Phi$ is presented below.

Lemma 3.5. Under conditions (5)-(7), (27) and (30), the continuous cocycle $\Phi$ associated with problem (21)-(24) is $\mathcal{D}$-pullback asymptotically compact in $H$, that is, for every $\tau \in \mathbf{R}, \omega \in \Omega$ and $B=\{B(\tau, \omega): \tau \in \mathbf{R}, \omega \in \Omega\} \in \mathcal{D}$, the sequence $\left\{\Phi\left(t_{n}, \tau-t_{n}, \theta_{-t_{n}} \omega,\left(u_{0, n}, v_{0, n}\right)\right)\right\}_{n=1}^{\infty}$ has a convergent subsequence in $H$ whenever $t_{n} \rightarrow \infty$ and $\left(u_{0, n}, v_{0, n}\right) \in B\left(\tau-t_{n}, \theta_{-t_{n}} \omega\right)$.

Proof. Since $t_{n} \rightarrow \infty, B \in \mathcal{D}$ and $\left(u_{0, n}, v_{0, n}\right) \in B\left(\tau-t_{n}, \theta_{-t_{n}} \omega\right)$, by (59), (76) and Lemma 4.1 we find that $\left\{\Phi_{2}\left(t_{n}, \tau-t_{n}, \theta_{-t_{n}} \omega,\left(u_{0, n}, v_{0, n}\right)\right)\right\}_{n=1}^{\infty}$ is bounded in $H$. Therefore, there exists $\left(\eta_{1}, \eta_{2}\right) \in H$ such that, up to a subsequence

$$
\Phi_{2}\left(t_{n}, \tau-t_{n}, \theta_{-t_{n}} \omega,\left(u_{0, n}, v_{0, n}\right)\right) \rightarrow\left(\eta_{1}, \eta_{2}\right) \text { weakly in } H .
$$

On the other hand, by Lemma 4.2 and Lemma 4.3, there exist $C=C(\tau, \omega)>0$ and $N=N(\tau, \omega, B)$ such that for all $n \geq N$

$$
\left\|\Phi_{2}\left(t_{n}, \tau-t_{n}, \theta_{-t_{n}} \omega,\left(u_{0, n}, v_{0, n}\right)\right)\right\|_{V}^{2} \leq C .
$$


By the compactness of embedding $V \hookrightarrow H$ and (77) we have, up to a subsequence,

$$
\Phi_{2}\left(t_{n}, \tau-t_{n}, \theta_{-t_{n}} \omega,\left(u_{0, n}, v_{0, n}\right)\right) \rightarrow\left(\eta_{1}, \eta_{2}\right) \text { strongly in } H,
$$

which implies that there exists $N_{1}=N_{1}(\tau, \omega, B, \varepsilon)>N$ such that for all $n \geq N_{1}$,

$$
\left\|\Phi_{2}\left(t_{n}, \tau-t_{n}, \theta_{-t_{n}} \omega,\left(u_{0, n}, v_{0, n}\right)\right)-\left(\eta_{1}, \eta_{2}\right)\right\|_{H}^{2} \leq \epsilon .
$$

By (59) and (76) we have

$\left\|\Phi\left(t_{n}, \tau-t_{n}, \theta_{-t_{n}} \omega,\left(u_{0, n}, v_{0, n}\right)\right)-\left(\eta_{1}, \eta_{2}\right)\right\|_{H}$

$\leq\left\|\Phi_{2}\left(t_{n}, \tau-t_{n}, \theta_{-t_{n}} \omega,\left(u_{0, n}, v_{0, n}\right)\right)-\left(\eta_{1}, \eta_{2}\right)\right\|_{H}+\left\|\Phi_{1}\left(t_{n}, \tau-t_{n}, \theta_{-t_{n}} \omega,\left(u_{0, n}, v_{0, n}\right)\right)\right\|_{H}$

$\leq\left\|\Phi_{2}\left(t_{n}, \tau-t_{n}, \theta_{-t_{n}} \omega,\left(u_{0, n}, v_{0, n}\right)\right)-\left(\eta_{1}, \eta_{2}\right)\right\|_{H}+\left\|v_{1}\left(\tau, \tau-t_{n}, \theta_{-\tau} \omega, v_{0, n}\right)\right\|_{L^{2}(\mathbf{U})}$

$\leq\left\|\Phi_{2}\left(t_{n}, \tau-t_{n}, \theta_{-t_{n}} \omega,\left(u_{0, n}, v_{0, n}\right)\right)-\left(\eta_{1}, \eta_{2}\right)\right\|_{H}+\mathrm{e}^{-\delta t_{n}}\left\|v_{0, n}\right\|_{L^{2}(\mathbf{U})}$,

which along with (80) implies

$$
\left\|\Phi\left(t_{n}, \tau-t_{n}, \theta_{-t_{n}} \omega,\left(u_{0, n}, v_{0, n}\right)\right)-\left(\eta_{1}, \eta_{2}\right)\right\|_{H} \rightarrow 0, t_{n} \rightarrow \infty,
$$

as desired. $\square$

Theorem 3.1. Suppose (5)-(7), (29) and (30) hold. Then the continuous cocycle $\Phi$ associated with problem (21)-(24) has a unique $\mathcal{D}$-pullback attractor $\mathcal{A}=\{\mathcal{A}(\tau, \omega): \tau \in \mathbf{R}, \omega \in \Omega\} \in \mathcal{D}$ in $H$.

Proof. Note that $\Phi$ is $\mathcal{D}$-pullback asymptotically compact in $H$ as demonstrated by Lemma 4.5 and has a closed measurable $\mathcal{D}$-pullback absorbing set by Lemma 4.4. Thus by proposition 2.1, we find that $\Phi$ has a unique $\mathcal{D}$-pullback random attractor $\mathcal{A}$ in $H . \square$

In conclusion, we prove the existence of a pullback random attractor in $H$ for the random dynamical system associated with the non-autonomous stochastic fractional FitzHugh-Nagumo system.

\section{Acknowledgements}

The authors would like to thank the reviewers for their helpful comments. This work was partially supported by the National Natural Science Foundation of China (Nos. 11771444, 11861013), the Yue Qi Young Scholar Project, China University of Mining and Technology (Beijing), Guangxi Natural Science Foundation (No. 2017GXNSFAA198221), Promotion of the Basic Capacity of Middle and Young Teachers in Guangxi Universities (No. 2017KY0340), the Fundamental Research Funds for the Central Universities, China University of Geosciences (Wuhan) (No. 2018061).

\section{Conflicts of Interest}

The authors declare no conflicts of interest regarding the publication of this paper. 


\section{References}

[1] FitzHugh, R. (1961) Impulses and Physiological States in Theoretical Models of Nerve Membrane. Biophysical Journal, 1, 445-466.

https://doi.org/10.1016/S0006-3495(61)86902-6

[2] Nagumo, J., Arimoto, S. and Yosimzawa, S. (1964) An Active Pulse Transmission Line Simulating Nerve Axon. Proceedings of the IRE, 50, 2061-2070.

https://doi.org/10.1109/JRPROC.1962.288235

[3] Ruelle, D. (1984) Characteristic Exponents for a Viscous Fluid Subjected to Time Dependent Forces. Communications in Mathematical Physics, 93, 285-300. https://doi.org/10.1007/BF01258529

[4] Marion, M. (1989) Finite-Dimensional Attractors Associated with Partly Dissipative Reaction-Diffusion Systems. SIAM Journal on Mathematical Analysis, 20, 816-844. https://doi.org/10.1137/0520057

[5] Marion, M. (1989) Inertial Manifolds Associated to Partly Dissipative ReactionDiffusion Systems. Journal of Mathematical Analysis and Applications, 143, 295-326. https://doi.org/10.1016/0022-247X(89)90043-7

[6] Shao, Z. (1998) Existence of Inertial Manifolds for Partly Dissipative Reaction Diffusion Systems in Higher Space Dimensions. Journal of Differential Equations, 144, 1-43. https://doi.org/10.1006/jdeq.1997.3383

[7] Gu, A. and Li, Y. (2014) Singleton Sets Random Attractor for Stochastic FitzHugh-Nagumo Lattice Equations Driven by Fractional Brownian Motions. Communications in Nonlinear Science and Numerical Simulation, 19, 3929-3937. https://doi.org/10.1016/j.cnsns.2014.04.005

[8] Huang, J. (2007) The Random Attractor of Stochastic FitzHugh-Nagumo Equations in an Infinite Lattice with White Noises. Physica D: Nonlinear Phenomena, 233, 83-94. https://doi.org/10.1016/j.physd.2007.06.008

[9] Li, Y. and Yin, J. (2016) A Modified Proof of Pullback Attractors in a Sobolev Space for Stochastic FitzHugh-Nagumo Equations. Discrete \& Continuous Dynamical Systems- $B, 21,1203-1223$.

[10] Wang, B. (2009) Random Attractors for the Stochastic FitzHugh-Nagumo System on Unbounded Domains. Nonlinear Analysis, 71, 2811-2828.

https://doi.org/10.1016/j.na.2009.01.131

[11] Liu, F., Turner, I., Anh, V., Yang, Q. and Burrage, K. (2013) A Numerical Method for the Fractional Fitzhugh-Nagumo Monodomain Model. ANZIAM Journal, 54, C608-C629. https://doi.org/10.21914/anziamj.v54i0.6372

[12] Lu, H., Bates, P.W., Lu, S. and Zhang, M. (2016) Dynamics of the 3D Fractional Ginzburg-Landau Equation with Multiplicative Noise on a Unbounded Domain. Communications in Mathematical Sciences, 14, 273-295. https://doi.org/10.4310/CMS.2016.v14.n1.a11

[13] Lu, H., Bates, P.W., Xin, J. and Zhang, M. (2015) Asymptotic Behavior of Stochastic Fractional Power Dissipative Equations on $R^{n}$. Nonlinear Analysis, 128, 176-198. https://doi.org/10.1016/j.na.2015.06.033

[14] Gu, A., Li, D., Wang, B. and Yang, H. (2018) Regularity of Random Attractors for Fractional Stochastic Reaction-Diffusion Equations on $R^{n}$. Journal of Differential Equations, 264, 7094-7137. https://doi.org/10.1016/j.jde.2018.02.011

[15] Wang, B. (2017) Asymptotic Behavior of Non-Autonomous Fractional Stochastic Reaction-Diffusion Equations. Nonlinear Analysis, 158, 60-82.

https://doi.org/10.1016/j.na.2017.04.006 
[16] Chepyzhov, V.V. and Vishik, M.I. (2002) Attractors for Equations of Mathematical Physics. AMS Colloquium Publications, Providence, RI. https://doi.org/10.1090/coll/049

[17] Temam, R. (1997) Infinite-Dimensional Dynamical Systems in Mechanics and Physics. In: Applied Mathematical Sciences, 2nd Edition, Volume 68, SpringerVerlag, New York.

[18] Arnold, L. (1998) Random Dynamical Systems. Springer-Verlag, New York. https://doi.org/10.1007/978-3-662-12878-7

[19] Bates, P.W., Lu, K. and Wang, B. (2009) Random Attractors for Stochastic Reaction-Diffusion Equations on Unbounded Domains. Journal of Differential Equations, 246, 845-869. https://doi.org/10.1016/j.jde.2008.05.017

[20] Crauel, H., Debussche, A. and Flandoli, F. (1997) Random Attractors. Journal of Dynamics and Differential Equations, 9, 307-341. https://doi.org/10.1007/BF02219225

[21] Crauel, H. and Flandoli, F. (1994) Attractors for Random Dynamical Systems. Probability Theory and Related Fields, 100, 365-393. https://doi.org/10.1007/BF01193705

[22] Di Nezza, E., Palatucci, G. and Valdinoci, E. (2012) Hitchhiker's Guide to the Fractional Sobolev Spaces. Bulletin des Sciences Mathématiques, 136, 521-573. https://doi.org/10.1016/j.bulsci.2011.12.004

[23] Servadei, R. and Valdinoci, E. (2014) On the Spectrum of Two Different Fractional Operators. Proceedings of the Royal Society of Edinburgh Section A: Mathematics, 144, 831-855. https://doi.org/10.1017/S0308210512001783

[24] Pazy, A. (1983) Semigroups of Linear Operators and Applications to Partial Differential Equations. In: Applied Mathematical Sciences, Volume 44, Springer-Verlag, New York. 